\title{
A Randomized Controlled Trial Comparing Clinical Outcomes and Toxicity of Lobaplatin- Versus Cisplatin-Based Concurrent Chemotherapy Plus Radiotherapy and High-Dose-Rate Brachytherapy for FIGO Stage II and III Cervical Cancer
}

\author{
Ji-Quan Wang, Tao Wang, Fan Shi, Yun-Yi Yang, Jin Su, Yan-Lan Chai, Zi Liu*
}

\begin{abstract}
Background: We designed this randomized controlled trial (RCT) to assess whether lobaplatin-based concurrent chemotherapy might be superior to cisplatin-based concurrent chemotherapy for FIGO stage II and III cervical cancer in terms of efficacy and safety. Materials and Methods: This prospective, open-label RCT aims to enroll 180 patients with FIGO stage II and III cervical cancer, randomly allocated to one of the three treatment groups (cisplatin $15 \mathrm{mg} / \mathrm{m}^{2}$, cisplatin $20 \mathrm{mg} / \mathrm{m}^{2}$ and lobaplatin $35 \mathrm{mg} / \mathrm{m}^{2}$ ), with 60 patients in each group. All patients will receive external beam irradiation (EBRT) and high-dose-rate intracavitary brachytherapy (HDR-ICBT). Patients in cisplatin $15 \mathrm{mg} / \mathrm{m}^{2}$ and $20 \mathrm{mg} / \mathrm{m}^{2}$ groups will be administered four cycles of $15 \mathrm{mg} / \mathrm{m}^{2}$ or $20 \mathrm{mg} / \mathrm{m}^{2}$ cisplatin intravenously once weekly from the second week to the fifth week during EBRT, while patients inthe lobaplatin $35 \mathrm{mg} / \mathrm{m}^{2}$ group will be administered two cycles of $35 \mathrm{mg} / \mathrm{m}^{2}$ lobaplatin intravenously in the second and fifth week respectively during pelvic EBRT. All participants will be followed up for at least 12 months. Complete remission rate and progression-free survival (PFS) will be the primary endpoints. Overall survival (OS), incidence of adverse events (AEs), and quality of life will be the secondary endpoints. Results: Between March 2013 and March 2014, a total of 61 patients with FIGO stage II and III cervical cancer were randomly assigned to cisplatin $15 \mathrm{mg} / \mathrm{m}^{2}$ group $(\mathrm{n}=21)$, cisplatin $20 \mathrm{mg} / \mathrm{m}^{2}$ group $(\mathrm{n}=21)$ and lobaplatin $35 \mathrm{mg} / \mathrm{m}^{2}$ group $(n=19)$. We conducted a preliminary analysis of the results. Similar rates of complete remission and grades 3-4 gastrointestinal reactions were observed for the three treatment groups $(P=0.801$ and 0.793 , respectively). Grade 3-4 hematologic toxicity was more frequent in the lobaplatin group than the cisplatin group. Conclusions: This proposed study will be the first RCT to evaluate whether lobaplatin-based chemoraiotherapy will have beneficial effects, compared with cisplatin-based chemoradiotherapy, on complete remission rate, PFS, OS, AEs and quality of life for FIGO stage II and III cervical cancer.
\end{abstract}

Keywords: FIGO stage II and III cervical cancer - RCT - lobaplatin - cisplatin - chemoradiotherapy

Asian Pac J Cancer Prev, 16 (14), 5957-5961

\section{Introduction}

Cervical cancer is second only to breast cancer as the most common female malignancy in both incidence and mortality worldwide (Seol et al., 2014). Platinum-based chemoradiotherapy has become an acceptable treatment for FIGO stage II and III disease. The platinum-based compounds cisplatin is among the most widely used and effective drugs. The activity of cisplatin-containing chemoradiotherapy in cervical cancer showed a reduction in the risk of recurrence of $40-60 \%$ (Whitney, et al., 1999). But there are approximately 275000 deaths annually because of treatment failure or recurrence of cervical cancer (Wiebe et al., 2012). Drug resistance to cisplatin is considered to be a major cause of treatment failure. Another problem for cisplatin, is its severe neuro and nephrotoxicity. This has led to the development of second- and third-generation platinum analogues, such as lobaplatin, with reduced toxicity and a better therapeutic index.

Lobaplatin (D-19466; 1, 2-diammino-methylcyclobutaneplatinum (II)-lactate) is a representative of the third-generation platinum compounds delivered as a diastereomeric mixture of S, S and R, R configurations of the carrier ligand, complex with DNA alkylating activity (Huang et al., 2013). It can obstruct the process of DNA replication and transcription by forming Pt-GG and Pt-AG intrachain cross-linking so as to interfere the running of tumor cell cycles (Eliopoulos et al., 1995). Compared with cisplatin, lobaplatin is considered to be less toxic, more soluble and stable in water and shows incomplete cross-resistance to cisplatin (McKeage et al., 


\section{Ji-Quan Wang et al}

2001; Deng et al., 2013). It has been approved in China for the treatment of chronic myelogenous leukaemia (CML), inoperable metastatic breast and small cell lung cancer. In addition, many clinical trials also suggest the effectiveness of lobaplatin in the treatment for various cancers, including esophageal, gastric, testicular and ovarian cancers (Harstrick et al., 1993). Now we conduct this randomized controlled trial (RCT) to compare clinical outcomes and toxicity of concomitant cisplatin versus lobaplatin plus radiotherapy and high-dose-rate intracavitary brachytherapy (HDR-ICBT) for FIGO stage II and III cervical cancer. This paper describes the trial design and analyzes the preliminary results. Based on the primary results, appropriate adjustments will be made for ongoing trials.

\section{Materials and Methods}

\section{Study design}

The aim of this study is to observe clinical outcomes and toxicity of concomitant cisplatin versus lobaplatin plus radiotherapy and HDR-ICBT for FIGO stage II and III cervical cancer. It was approved by the Ethics Committee of The First Affiliated Hospital of Xi'an Jiaotong University. All study participants provided written informed consent before participation.

This clinical trial was a prospective open-label RCT at The First Affiliated Hospital of Xi' an Jiaotong University. It aimed to enroll 180 patients with FIGO stage II and III cervical cancer who meet the study criteria below. Using a random-number table (Center of Evidence-Based Medicine and Clinical Epidemiology, The First Affiliated Hospital of Xi' an Jiaotong University), patients would be randomly assigned in a 1:1:1 ratio to either the group 1,2 or 3 (Figure 1). The primary endpoint with respect to efficacy is complete remission rate and progression-free survival (PFS). Secondary endpoints are overall survival (OS),

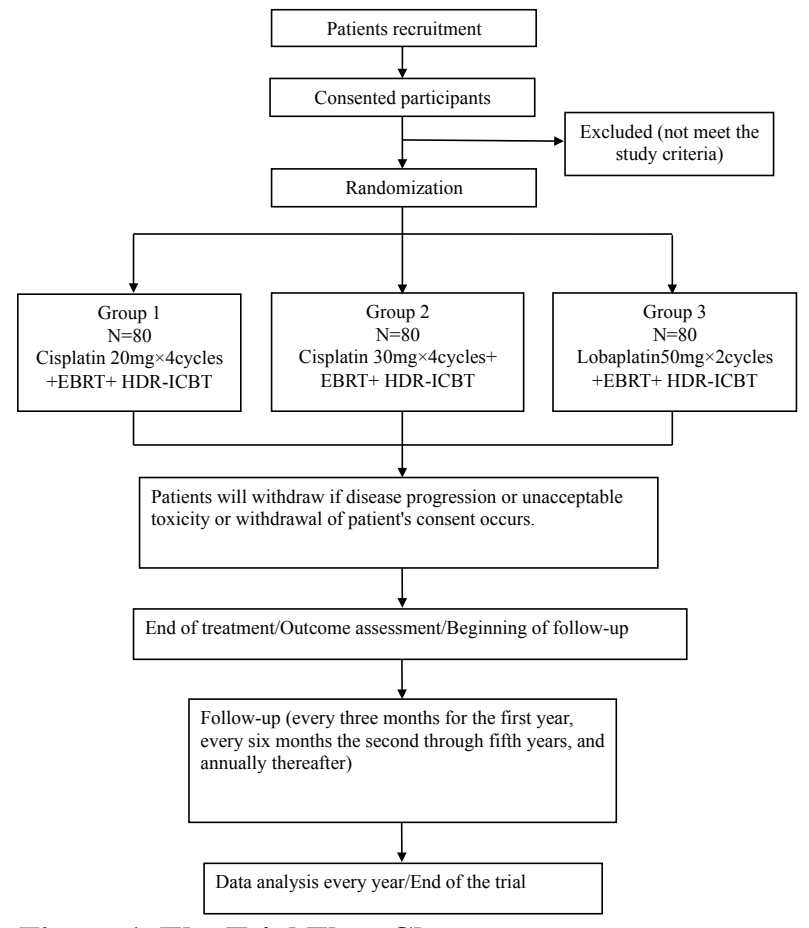

Figure 1. The Trial Flow Chart incidence of adverse events, and quality of life (QOL).

Patient registration began on March 2013 and is to continue for three years or until 180 individuals have been randomly assigned. When all the patients will have been followed up for at least 12 months, the full study is expected to be finished.

\section{Patients}

Inclusion criteria included (1) hospitalized patients, age 65years; (2) Eastern Cooperative Oncology Group (ECOG) performance status of 0 to 2; (3) pathological diagnosis of cervical cancer, FIGO stage II and III disease by pelvic examinations; (3) no previous treatment with chemotherapy or radiotherapy for cancer; (4) hematology, liver and kidney function are normal; (5) Good understanding and compliance by patients with the pilot program, and provision of informed consent.

Baseline examinations included physical status, physical examination (height, weight, body surface area, pelvic examinations and palpation of superficial lymph nodes), computed tomography (CT) of the chest and abdomen, magnetic resonance imaging (MRI) of the pelvis, electrocardiography (ECG), complete blood count and biochemistry panels.

\section{Treatment}

Radiotherapy: All the patients received platinum-based chemoradiotherapy. Radiotherapy included external beam irradiation (EBRT) and HDR-ICBT. EBRT was implanted by a linear accelerator of three-dimensional conformal radiation therapy (3D-CRT) or intensity-modulated radiation therapy (IMRT). According to Radiation Therapy Oncology Group (RTOG) guidelines (Small et al., 2008), the clinical target volume (CTV) included the common, external, and internal iliac lymph node regions and the upper $3.0 \mathrm{~cm}$ of the vagina. The superior margin of the external radiation field was located at the abdominal aortic bifurcation, went down along $7 \mathrm{~mm}$ outside the iliac vessels and the inferior border was determined by the degree of vaginal violations. External irradiation was delivered to the whole pelvis ( 2 Gy per fraction), with five fractions administered per week for a total of 25 fractions and 50 Gy. After completing external irradiation, gynecological examinations were performed to determine the appropriate ICBT program and dose. ICBT was performed using the Fletcher-Suit-Delclos set with a microSelectron HDR (Nucletron, Veenendaal, Netherlands). The total planned dose to point A for HDR-ICBT was $24 \mathrm{~Gy}$ in four fractions.

\section{Chemotherapy}

Patients in group 1 were administered four cycles of $15 \mathrm{mg} / \mathrm{m}^{2}$ cisplatin intravenously once weekly from the second week to the fifth week during EBRT. Those in group 2 received four cycles of $20 \mathrm{mg} / \mathrm{m}^{2}$ cisplatin intravenously once weekly from the second to fifth week during EBRT. Patients in group 3 were administered two cycles of 35 $\mathrm{mg} / \mathrm{m}^{2}$ lobaplatin intravenously in the second and fifth week respectively during pelvic EBRT. All patients were administered antiemetic drugs prior to chemotherapy. Physical status and routine blood should be performed weekly during the treatment. If bone marrow suppression 
occurred, appropriate and timely interventions should be taken. The patients with III-IV grade thrombocytopenia or leukopenia should be treated with recombinant human interleukin-11 or recombinant human granulocyte colonystimulating factor. The treatment would be stopped if disease progression or unacceptable toxicity or withdrawal of patient's consent occurs.

\section{Follow-up}

Follow-up will consist of a telephone survey and a visit to the clinic for re-examination. Patients were re-examined every three months for the first year, every six months the second through fifth years, and annually thereafter. Gynecologic examination and supraclavicular lymph node palpation were performed at each appointment. Chest $\mathrm{X}$-rays were obtained one year after treatment. Suspected cases of persistent or recurrent disease were confirmed by biopsy whenever possible. For these cases, chest CT and abdomino-pelvic CT or MRI were obtained to detect the site of failure.

\section{Definition of early outcomes and toxicity}

Tumor evaluations were performed at entry and after treatment by pelvic examinations according to Response Evaluation Criteria in Solid Tumors (RECIST)/WHO evaluation criteria (Tsuchida et al., 2001). A complete response (CR) was defined as a disappearance of all evidence of the tumor and no development of new lesions for at least 4 weeks. A partial response (PR) was defined as a decrease of at least $50 \%$ in the sum of the products of the largest perpendicular diameters of all measurable lesions. The severity of the complications associated with chemotherapy was classified according to the National Cancer Institute Common Toxicity Criteria (NCI-CTCv2.0) (Hughes et al., 2008).

\section{Statistical analysis}

Differences between the two treatment groups were assessed using a $\chi^{2}$ test or Fisher's exact test for categorical variables. An independent sample t-test was used for continuous variables. A P-value less than 0.05 were considered statistically significant. Statistical Package for Social Scientists (SPSS, version 18.0, IL) was used for all analyses.

\section{Trial status}

As of March 2014,61 patients have been enrolled and randomized for this trial, and recruitment is ongoing. Now we conduct a preliminary analysis of the results.

\section{Results}

\section{Patient characteristics}

Between March 2013 and March 2014, sixty-one patients were randomly allocated to group 1, 2 and 3 . No patients withdraw from the trail and all 61 patients entered a preliminary analysis of results. Of the 61 eligible patients, 21, 21 and 19 cases were randomly assigned to group 1, 2 and 3. Baseline characteristics turned out to be well balanced between the two groups with no significant imbalances in age, maximum tumor diameter, FIGO stage, pathological grading and ERBT methods. Baseline characteristics are shown in Table 1.

\section{Efficacy}

Complete remission rate was $52.38 \%, 47.62 \%$ and $42.11 \%$ for group 1, 2 and 3 (Table 2), which did not significantly differ between the three treatment groups $(\mathrm{P}=0.801)$. No distant metastases occurred in any patient when we evaluated for efficacy.

\section{Adverse effects}

All patients were evaluable for toxicity assessments. The most common grades 3-4 adverse events (AEs) are summarized in Table 3.

The incidence of grades 3-4 gastrointestinal reactions was similar for the three treatment groups $(\mathrm{P}=0.793)$. Grades 3-4 hematologic AEs were more frequent in

Table 1. Patient Characteristics

\begin{tabular}{|c|c|c|c|c|}
\hline Characteristic, n (\%) & $\begin{array}{l}\text { Group } 1 \\
(\mathrm{n}=21)\end{array}$ & $\begin{array}{c}\text { Group } 2 \\
(\mathrm{n}=21)\end{array}$ & $\begin{array}{c}\text { Group } 3 \\
(n=19)\end{array}$ & $P$-value \\
\hline$\overline{\text { Age }}$ & & & & 0.136 \\
\hline$\leq 50$ & $15(71.43)$ & $10(47.62)$ & $8(42.11)$ & \\
\hline$>50$ & $6(28.57)$ & $11(52.38)$ & $11(57.89)$ & \\
\hline Pathological grading & & & & 0.383 \\
\hline 1 & $1(4.76)$ & $1(4.76)$ & $0(0)$ & \\
\hline 2 & $20(95.24)$ & $18(85.71)$ & $16(84.21)$ & \\
\hline 3 & $0(0)$ & $2(9.52)$ & $3(15.79)$ & \\
\hline Maximum tumor diameter (mm) & & & & 0.445 \\
\hline$>40$ & $5(23.81)$ & $2(9.52)$ & $4(21.05)$ & \\
\hline$\leq 40$ & $16(76.19)$ & $19(90.48)$ & $15(78.95)$ & \\
\hline FIGO stage & & & & 0.877 \\
\hline IIA & $1(4.76)$ & $0(0)$ & $1(5.26)$ & \\
\hline IIB & $11(52.38)$ & $12(57.14)$ & $9(47.37)$ & \\
\hline IIIA & $1(4.76)$ & $1(4.76)$ & $0(0)$ & \\
\hline IIIB & $8(38.10)$ & $8(38.10)$ & $9(47.37)$ & \\
\hline ERBT methods & & & & 0.627 \\
\hline 3DCRT & 10 & 12 & 8 & \\
\hline IMRT & 11 & 9 & 11 & \\
\hline
\end{tabular}




\section{Table 2. Response Evaluation}

\begin{tabular}{lccc}
\hline Response, $n(\%)$ & Group1(n=21) & Group2(n=21) & Group3(n=19) \\
\hline CR & $11(52.38)$ & $10(47.62)$ & $8(42.11)$ \\
PR & $10(47.62)$ & $11(52.38)$ & $11(57.89)$ \\
\hline
\end{tabular}

$* P=0.801$ for the three treatment considering $\mathrm{CR}$.

Table 3. Grades 3 and 4 Acute Toxicities

\begin{tabular}{lcccc}
\hline $\begin{array}{l}\text { Adverse events } \\
\mathrm{n}(\%)\end{array}$ & $\begin{array}{c}\text { Group1 } \\
(\mathrm{n}=21)\end{array}$ & $\begin{array}{c}\text { Group2 } \\
(\mathrm{n}=21)\end{array}$ & $\begin{array}{r}\text { Group3 } \\
(\mathrm{n}=19)\end{array}$ & $\begin{array}{c}P \\
\text { value }\end{array}$ \\
\hline Gastrointestinal reactions & $1(4.76)$ & $2(9.52)$ & $1(5.26)$ & 0.793 \\
Leukocytopenia & $3(14.29)$ & $5(23.81)$ & $11(57.89)$ & 0.008 \\
Thrombocytopenia & $0(0)$ & $0(0)$ & $6(31.58)$ & 0.001 \\
\hline
\end{tabular}

group 3 than group 1 and 2 (leucopenia: 14.29 vs 23.81 vs $57.89 \%(\mathrm{P}=0.008)$; thrombocytopenia: 0 vs 0 vs $31.58 \%$ $(P=0.001))$.

The incidence of grades 3-4 leukocytopenia and gastrointestinal reactions was higher in group 2 than group 1 , but the differences did not reach statistical significance (leukocytopenia: 14.29 vs $23.81, \mathrm{P}=0.697$; gastrointestinal reactions: 4.76 vs $9.52 \%, P=1.000)$.

\section{Treatment duration}

Treatment duration of the three groups is 48.04,47.66 and 50.05 days, respectively, with no significant difference $(p=0.115)$.

\section{Discussion}

This study is the first randomized control trial to investigate the efficacy and toxicity of lobaplatin combined with concurrent radical radiation for cervical cancer. Although cisplatin-based chemoradiotherapy is a promising option for FIGO stage II and III disease, $15-30 \%$ patients will relapse and metastasis. Lobaplatin, characterized by no crossing drug resistance with other platinum-based drugs, good water solubility, broad anti-tumor spectrum, strong anti-tumor activity and low toxicity, exerts definite effects in the treatment of various tumors, such as breast cancer (Engel et al., 2012; Deng et al., 2013), lung cancer (Xie et al., 2012), esophageal carcinoma, gastrointestinal cancer (Wang et al., 2014; Zhao et al., 2014) and malignant pleural effusion and ascites (Huang et al., 2013). In terms of cervical cancer, the in-vitro experimental studies have demonstrated that lobaplatin inhibits cell proliferations in human cervical cancer CaSki cells by inducing apoptosis, cell cycle arrest and changing many kinds of protein molecule expression level (Li et al., 2014). The dose-limiting toxicity of lobaplatin is thrombocytopenia, with incidence ranging from $14.5 \%$ to $26 \%$ and a nadir at approximately 2 weeks after drug administration (Degardin et al., 1995; Welink et al., 1999; Zhao et al., 2014). Leukopenia is less severe than thrombocytopenia, and the drug does not induce nephrotoxicity, neurotoxicity, or ototoxicity (Welink et al., 1999).

The primary outcome of our RCT indicates that complete remission rate was comparable between cisplatinand lobaplatin-based chemoradiotherapy for FIGO stage II and III cervical cancer, ranging from $42.11 \%$ to $52.38 \%$.
The adverse reactions mainly include thrombocytopenia, leukopenia and gastrointestinal toxicity. The incidence of thrombocytopenia and leukopenia in lobaplatin-based chemoradiotherapy group is significantly higher than that in cisplatin-based chemoradiotherapy group. Even so, treatment duration of lobaplatin-based chemoradiotherapy group was similar to cisplatin-based chemoradiotherapy group. There is no standard dose for lobaplatin combined with radical radiotherapy for cervical cancer. Climbing test should be implemented in order to seek the optimal dose. Based on our preliminary results, the dose of lobaplatin will be reduced to $30 \mathrm{mg} / \mathrm{m}^{2}$. Efficacy and side effects are comparable between cisplatin $15 \mathrm{mg} / \mathrm{m}^{2}$ group and $20 \mathrm{mg} /$ $\mathrm{m}^{2}$ group, which indicate $15 \mathrm{mg} / \mathrm{m}^{2}$ is the optimal dose of cisplatin combined with radical radiotherapy for cervical cancer.

In conclusion, efficacy of lobaplatin- and cisplatinbased chemoradiotherapy is comparable while thrombocytopenia and leucopenia of lobaplatin-based chemoradiotherapy are higher than cisplatin-based chemoradiotherapy according to preliminary results of our RCT. The final results and long-term efficacy need to be further observed and analyzed.

\section{Acknowledgements}

This study was financially supported by the Department of Health Key Program of Shaanxi Province (No. 2010A02). We are grateful to our coworkers in department of radiotherapy oncology of First Affiliated Hospital of $\mathrm{Xi}$ 'an Jiaotong University for their support in acquisition of data and clinical observation. We also thank the patients for participating in this study.

\section{References}

Degardin M, Armand JP, Chevallier B, et al (1995). A clinical screening cooperative group phase II evaluation of lobaplatin (ASTA D-19466) in advanced head and neck cancer. Investigational New Drugs, 13, 253-5.

Deng QQ, Huang XE, Ye LH, et al (2013). Phase II trial of Loubo ${ }^{\circledR}($ Lobaplatin) and pemetrexed for patients with metastatic breast cancer not responding to anthracycline or taxanes. Asian Pac J Cancer Prev, 14, 413-7.

Eliopoulos AG, Kerr DJ, Maurer HR, Hilgard P, Spandidos DA (1995). Induction of the c-myc but not the $\mathrm{cH}$-ras promoter by platinum compounds. Biochem Pharmacol, 50,33-8.

Engel JB, Martens T, Hahne JC, et al (2012). Effects of lobaplatin as a single agent and in combination with TRAIL on the growth of triple-negative p53-mutated breast cancers in vitro. Anti-Cancer Drugs, 23, 426-36.

Ferlay J, Soerjomataram I, Ervik M, et al (2013). GLOBOCAN 2012 v1.0, Cancer Incidence and Mortality Worldwide. In IARC CancerBase No. 11 (Internet). Lyon, France, International Agency for Research on Cancer. Available from, http://globocan.iarc.fr, accessed on day/month/year.

Harstrick A, Bokemeyer C, Scharnofkse M, et al (1993). Preclinical activity of a new platinum analogue, lobaplatin, in cisplatin-sensitive and resistant human testicular, ovarian and gastric carcinoma cell lines. Cancer Chemother Pharmacol, 33, 43-7.

Huang XE, Wei GL, Huo JG, et al (2013). Intrapleural or intraperitoneal lobaplatin for treatment of patients with malignant pleural effusion or ascites. Asian Pac J Cancer 
Prev, 14, 2611-4.

Hughes R (2008). NCI-CTC vs TNS, which tool is better for grading the severity of chemotherapy-induced peripheral neuropathy? Nature Clinical Practice Neurology, 4, 68-69.

Li X, Ran L, Fang W, Wang D (2014). Lobaplatin arrests cell cycle progression, induces apoptosis and alters the proteome in human cervical cancer cell Line CaSki. Biomed Pharmacoth, 68, 291-7.

McKeage M J (2001). Lobaplatin, a new antitumour platinum drug. Expert opinion on investigational drugs, 10, 119-28.

NCCN, Practice Guidelines in Oncology. 2013. http://www.nccn. org/professionals/physician_gls/f_guidelines.asp.

Small W Jr, Mell LK, Anderson P, et al (2008). Consensus guidelines for delineation of clinical target volume for Intensity-Modulated pelvic radiotherapy in postoperative treatment of endometrial and cervical cancer. Int J Radiat Oncol Biol Phys, 71, 428-34.

Tsuchida Y, Therasse P (2001). Response evaluation criteria in solid tumors (RECIST), new guidelines. Med Pediatr Oncol, 37,1-3.

Wang N, Lv YZ, Xu AH, Huang YR, Peng L, Li JR (2014). Application of lobaplatin in trans-catheter arterial chemoembolization for primary hepatic carcinoma. Asian Pac J Cancer Prev, 15, 647-650.

Welink J, Boven E, Vermorken JB, Gall HE, van der Vijgh WJ (1999). Pharmacokinetics and pharmacodynamics of lobaplatin (D-19466) in patients with advanced solid tumors, including patients with impaired renal or liver function. Clin Cancer Res, 5, 2349-58.

Whitney CW, Sause W, Bundy BN, et al (1999). Randomized comparison of fluorouracil plus cisplatin versus hydroxyurea as an adjunct to radiation therapy in Stage IIB-IVA carcinoma of the cervix with negative para-aortic lymph nodes, a gynecologic oncology group and southwest oncology group study. J Clin Oncol, 17, 1339-48.

Wiebe E, Denny L, Thomas G (2012). Cancer of the cervix uteri. Int J Gynaecol Obstet, 119, 100-9.

Xie CY, Xu YP, Jin W, Lou LG (2012). Antitumor activity of lobaplatin alone or in combination with antitubulin agents in non-small-cell lung cancer. Anticancer Drugs, 23, 698-705.

Zhao C, Wang XJ, Wang S, et al (2014). Lobaplatin Combined Floxuridine/Pirarubicin-based Transcatheter Hepatic Arterial Chemoembolization for Unresectable Primary Hepatocellular Carcinoma. Asian Pac J Cancer Prev, 15, 2057. 